\title{
Desarrollo de proceso para elaboración de horma personalizada mediante el uso de herramientas de manufactura flexible. Una visión sistémica.
}

\author{
Neri Ledezma, Sergio ${ }^{a}$; Santana-Madrigal, Gloria ${ }^{\mathrm{b}}$ \& García-Alvarez, Norberto $^{\mathrm{c}}$ \\ ${ }^{a}$ Profesor Investigador (Departamento de Diseño Industrial, Universidad Politécnica de la Zona Metropolitana de \\ Guadalajara, Guadalajara, México),nerixx@hotmail.com \\ ${ }^{b}$ Profesor Investigador (Departamento de Terapia Física, Universidad Politécnica de la Zona Metropolitana de \\ Guadalajara, Guadalajara, México), gsantana1@hotmail.com \\ ${ }^{\mathrm{c}}$ Profesor Cátedra (Departamento de Diseño Industrial, Universidad Politécnica de la Zona Metropolitana de \\ Guadalajara, Guadalajara, México),nogaal74@hotmail.com
}

\begin{abstract}
Resumen
El presente proyecto busca la generación de un nuevo sistema para el desarrollo de componentes en la elaboración de zapatos mediante la implementación de sistemas computacionales para el diseño, desarrollo, evaluación y fabricación del producto. Particularmente se busca el desarrollo de hormas para menores con Malformaciones Congénitas (MC), que permita a través de su elaboración, la eventual confección de calzado personalizado ajustado a la morfología de las extremidades. El objetivo e intención del presente proyecto, busca atender la malformación de pie equino varo aducto congénito (PEVAC); alteración que compromete el eje transversal del pie, permitiendo con ello, coadyuvar a las técnicas terapéuticas convencionales para el tratamiento de este tipo de anomalías estructurales y/o funcionales.

La metodología utilizada para tal fin, se basa en la aplicación de las herramientas de manufactura flexible (escáner $3 D$, digitalizador, software CAD, CAE e impresión $3 D$ ) para el desarrollo de productos industriales. El resultado obtenido, es un par de hormas adecuadas a la malformación referida, generando una posibilidad importante de abrir un nicho de mercado en el desarrollo y comercialización de este tipo de insumos que aportan valor a un sector industrial todavía tradicional; método y producto que permiten mejorar la calidad de vida de las personas y su inclusión en la sociedad.
\end{abstract}

Palabras clave: Hormas calzado, impresión $3 d$, pié equino varo abducto congénito. 
Desarrollo de proceso para elaboración de horma personalizada mediante el uso de herramientas de manufactura flexible. Una visión sistémica,

\begin{abstract}
This project aims to create a new system for the development of components in the production of shoes by implementing computer systems for the design, development, testing and manufacturing. Particularly sought is the development of lasts for children with Congenital Malformations (MC), which through its elaboration, allows eventually the making of custom shoes adjusted to the morphology of the extremities. The purpose and intent of this project seeks to address the malformation of congenital clubfoot adduct; alteration compromising the transverse axis of the foot, thereby permitting, contribute to conventional therapeutic techniques for the treatment of this type of structural anomalies and functionality.

The methodology used for this purpose is based on the implementation of flexible manufacturing tools ( $3 D$ scanner, digitiser, software CAD, CAE and $3 D$ printing) for the development of industrial products. The result is a pair of appropriate malformation referred lasts, generating a significant possibility of opening a niche in the development and marketing of this type of inputs that add value to a still traditional industrial sector; method and product that improve the quality of life of people and their inclusion in society.
\end{abstract}

Key words: Shoe lasts, $3 D$ printing, clubfoot

\title{
1. Estado del arte
}

Desde hace cientos de años, las hormas han servido como réplica del pie humano para la elaboración de calzado, ya sea de madera o de plástico, las hormas tienen la finalidad de sustituir el pie durante la fabricación del calzado para servir como superficie de trabajo, sobre la cual, se agregan y ensamblan componentes que permitirán dar forma y estilo al mismo.

El segundo objetivo consiste en reflejar la orientación de la moda y los requisitos estéticos: es decir, mostrar una forma perfecta. A partir del modelo de zapato elegido, las hormas definen el volumen interior y la forma exterior del zapato elaborado, de acuerdo a las medidas que se han tomado del pie y en base a la morfología que éstos tengan.

Hasta hace algunas décadas, la fabricación de zapatos para cualquier tipología de persona se podría llevar a cabo de manera personalizada; los artesanos de calzado tenían la capacidad y experiencia para llevar a cabo las adecuaciones de hormas para realizar una copia abstracta del pie humano, elemento cuantitativo que suponía un alto valor agregado, ya que los zapatos una vez confeccionados calzaban perfectamente en el pie del cliente, cualidad que le confería al artesano una ventaja competitiva singular por encima de los fabricantes de calzado estandarizado.

El sector del calzado está compuesto por diferentes tipos de clientes y usuarios que tienen diversas necesidades, las cuales se convierten finalmente en criterios de diseño y producción. Tradicionalmente, estos grupos de criterios han tratado de solucionar las necesidades de los segmentos de la población más amplios, dejando de lado las necesidades de grupos de personas quienes por alguna razón, han nacido con 
alguna malformación que les impide entrar en los estándares de un calzado convencional. La fabricación de productos personalizados se ha configurado como la fuerza impulsora para muchas industrias, especialmente para las relacionadas con la moda, donde se están intentando implementar varias alternativas y métodos para abordar la personalización en masa como es el caso de la empresa Adidas (Adidas, 2015) con sus runner deportivos impresos en 3D. Sin embargo, este tipo de productos, sigue privilegiando los pies "sanos" marginando a la población que cuenta con una deformación.

La fabricación convencional de hormas se realiza en principio mediante la configuración de las características volumétricas y dimensionales a través de software de diseño asistido por computadora (CAD por sus siglas en inglés), información que es procesada por un software de manufactura asistida por computadora (CAM por sus siglas en inglés) para la generación del código "G", fichero electrónico que describirá las secuencias de operación de equipos de control numérico por computadora $(\mathrm{CNC})$ para la operación de equipos de desbaste, las cuales llevarán cabo el proceso de transformación física de los bloques de poliestireno de alta densidad para la obtención de la horma. - CAD)

Las herramientas de manufactura flexible (HMF), entendidas desde el punto de vista del uso de tecnologías sistémicas que permiten la generación de productos complejos y altamente personalizados, han sido incorporadas en sectores no convencionales como las ciencias médicas, obteniendo resultados importantes en los campos de las prótesis y ortesis a través del uso de escáner tridimensionales y digitalizadores, los cuales permiten la obtención de modelos virtuales de geometrías corporales, información que a través de su edición por sistemas $\mathrm{CAD}$, permiten la obtención de ficheros electrónicos imprimibles en sistemas de manufactura aditiva o prototipado rápido (PR); equipos de impresión en $3 \mathrm{D}$ que de acuerdo a los parámetros de calidad y tipo de material, generan productos fiables para el uso corporal cotidiano.

El pie equino varo aducto congénito (PEVAC), es una malformación musculo esquelética común. Ciertamente hay factores etiológicos que no han sido bien entendidos. (Matthew B. Dobbs, 2009) Se sabe que puede ser multifactorial y no existen reportes del padecimiento de manera idiopática en productos menores a las siete semanas de gestación (Vázquez 1987). En su gran mayoría se presentan de manera esporádica, sin embargo existen elementos asociados que se relacionan con la deformidad y que son bien conocidos (historia familiar, genética, factores estacionales, factores mecánicos intrauterinos, miogénesis anormal, causas neuromusculares entre otras) (Staheli, 2006)

El PEVAC es de las malformaciones musculo esqueléticas más comunes; los reportes de prevalencia van desde 0.7 hasta 6.8 por cada 1,000 nacidos vivos. Tomando como base la tasa de nacimientos en México, se estima que al año hay 5,600 nuevos casos de esta deformidad (Torres, 2010). Los problemas ortopédicos del pie en este tipo de niños difícilmente son devueltos y recuperados a su posición y/o forma natural, obligando a usar un calzado que permita además de la protección, un soporte adecuado que posibilite emprender la marcha con el menor daño en la estructura del pie. 
Desarrollo de proceso para elaboración de horma personalizada mediante el uso de herramientas de manufactura flexible. Una visión sistémica,

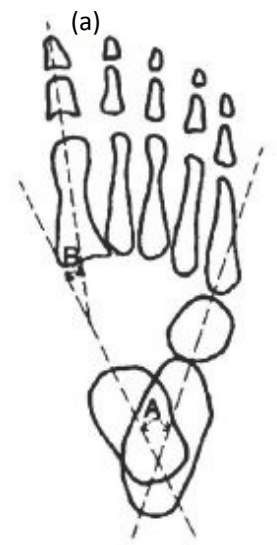

(b)

Fig. 1 Vista superior (a) pie normal, (b) pie deforme. Fuente: E. Vijayaragavan (2014)

\section{Material y métodos}

Para el desarrollo del proyecto se llevaron a cabo investigación de tipo documental y de campo, en las que se obtuvo información de tipo cuantitativa respecto a las características a cumplir por parte de la horma una vez impresa, y en segundo término, en relación al padecimiento PEVAC sobre un caso real: niña de 9 años con PEVAC que ha sido intervenida quirúrgicamente para la extensión de su tendón de Aquiles.

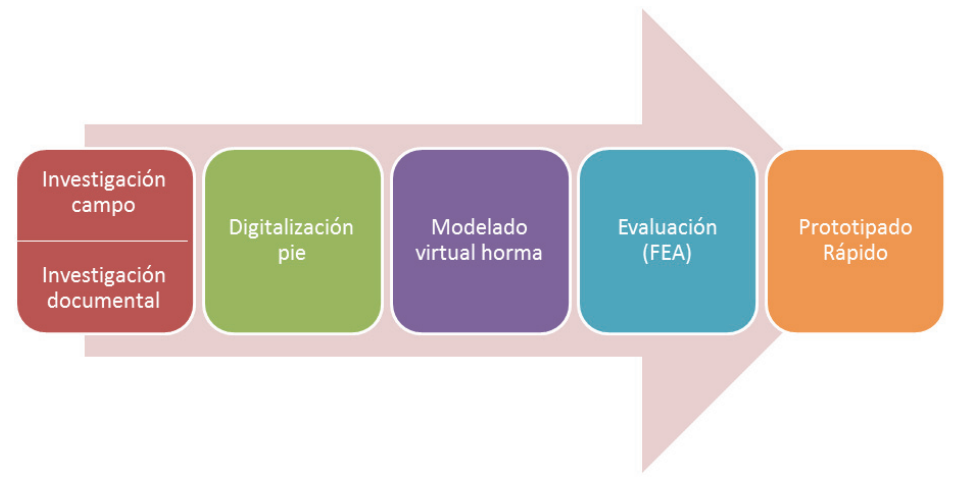

Fig. 2 Método de trabajo. Fuente: Elaboración propia (2016)

Referente a la investigación de campo, se identificaron los esfuerzos y tensiones de la horma durante el proceso de fabricación del calzado en la empresa Diseños y accesorios S. A. de C.V., empresa de tamaño mediano (245 trabajadores) ubicada en la Cd. De Guadalajara Jalisco, México, cuya fabricación promedio es de 2,000-2,500 pares semanales.

Los esfuerzos se documentaron en base a las lecturas de cada uno de los equipos y procesos más significativos que se emplean en la fabricación del calzado, mismos que someten a la horma a diversos esfuerzos y compresiones durante el armado de sus componentes de acuerdo a como se muestra en la figura no. 3 . 


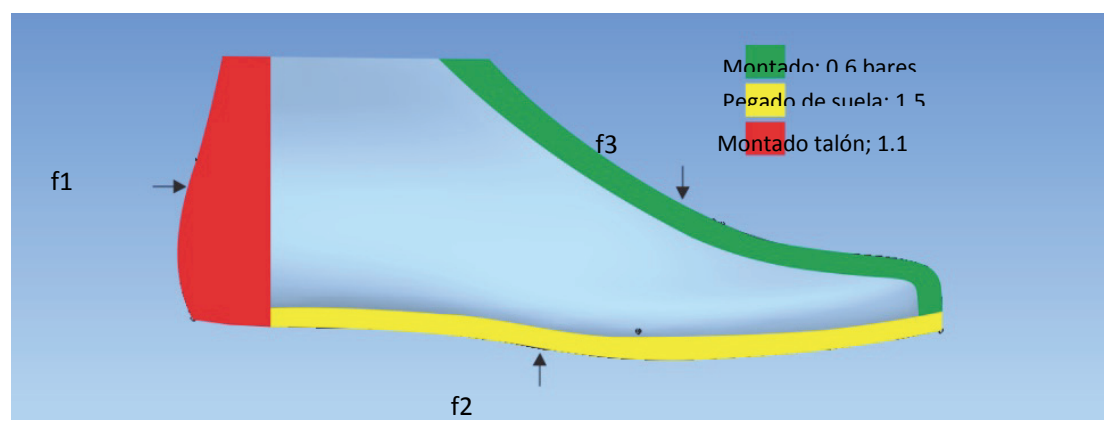

Fig. 3 Distribución de esfuerzos por proceso.

Fuente: Elaboración propia (2016)

Respecto a la digitalización de los pies, se generó un molde elaborado por la superposición de capas de vendas de algodón recubierta de una capa de yeso grado médico para cada pie tal como se muestra en la figura número 4. Una vez fraguado el molde y retirado del pie se vertió sulfato de calcio (yeso cerámico) para la obtención de las réplicas.

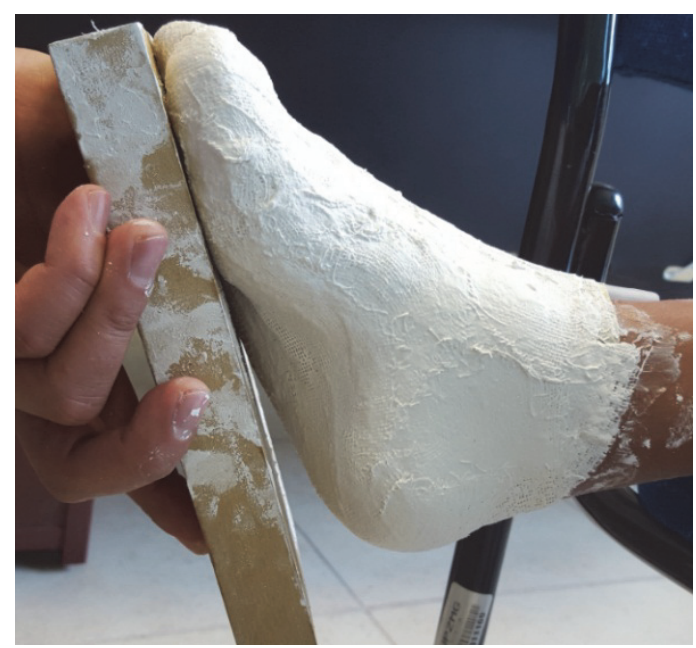

Fig. 4 Moldes de yeso cerámico. Fuente: Elaboración propia (2016)

Posteriormente fueron sometidos al proceso de digitalización mediante el uso de un brazo digitalizador marca MicroScribe con una precisión de $+/-0.0508 \mathrm{~mm}$. La captura de la geometría se realizó mediante la implementación de un software CAD Rhinoceros versión 5.0 tal como se muestra en la figura número 5. 
Desarrollo de proceso para elaboración de horma personalizada mediante el uso de herramientas de manufactura flexible. Una visión sistémica,

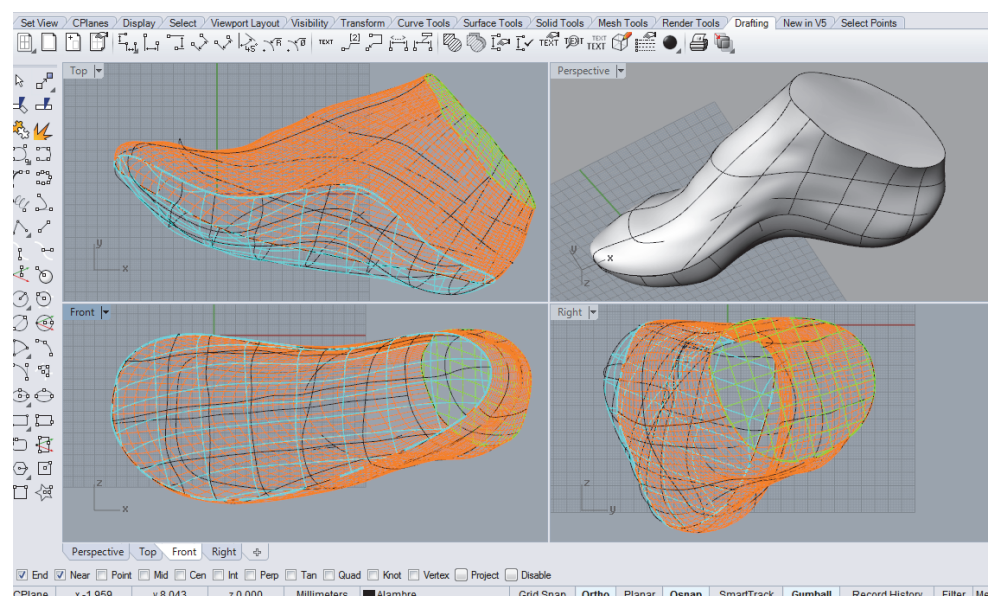

Fig. 5 Digitalización del pie. Vistas generales. Rhino V 5.0. Fuente: Elaboración propia (2016)

A partir de la obtención de la geometría del pie se desarrolló la horma personalizada en base a la utilización de método AK64 a través del cual se determinaron los siguientes parámetros para la configuración de la horma: longitud total (talla), recio, cintura, empeine, ancho de plantilla interno, ancho de plantilla externo, ángulo de dedo gordo, ángulo de dedo chico, ángulo de talón y grueso de punta. En referencia al spring (altura de la punta respecto al suelo) y al tipo de punta se consideraron en base a la tendencia actual.

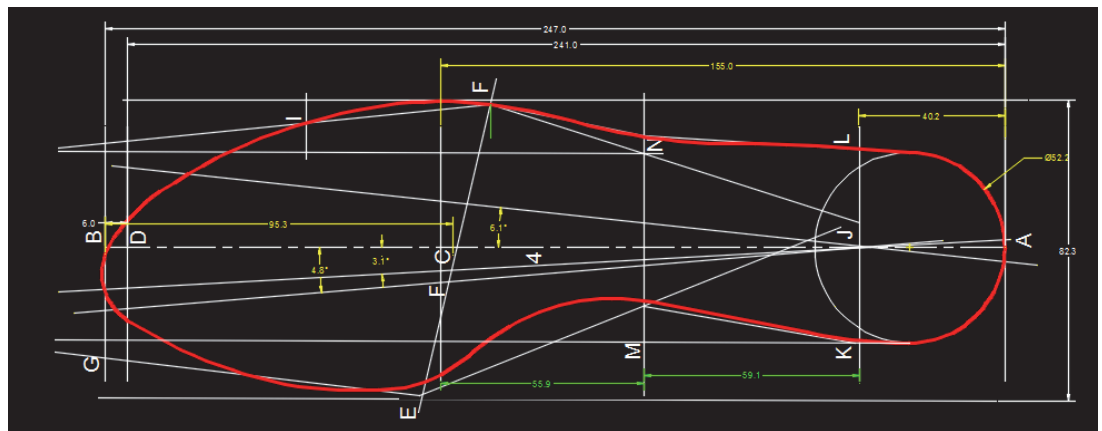

Fig. 6 Desarrollo de planta de horma mediante la aplicación de método AK64. AutoCad 2016 - Autodesk.. Fuente: Elaboración propia (2016)

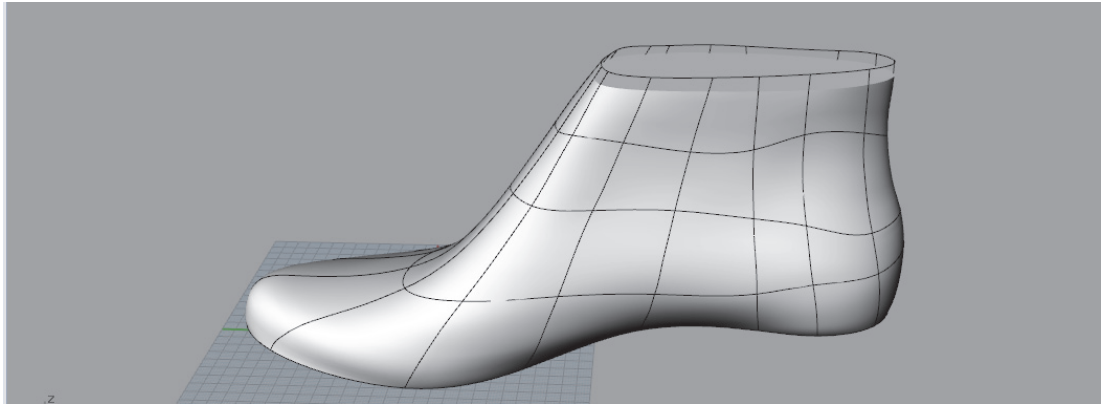

Fig. 7 Desarrollo volumétrico de horma en Rhino V 5.0. Fuente: Elaboración propia (2016) 
La factibilidad técnica de la horma se comprobó mediante la elaboración de una simulación de esfuerzos a través del uso de módulo de software CAE de análisis por elemento finito (FEA por sus siglas en inglés) considerando los esfuerzos a los que se somete la horma durante las distintas fases de su proceso de elaboración. Para tal efecto se consideró una estructura interna de tipo romboide de $10 \mathrm{~mm}$ de lado con espesor de pared de $0.5 \mathrm{~mm}$ y una capa exterior de $0.5 \mathrm{~mm}$. El material considerado fue el acrílonitrilo butadieno estireno (ABS) con un módulo de tracción de $2.1-2.4 \mathrm{GPa}$. Los esfuerzos tangenciales a los que se sometió la horma fueron $15 \mathrm{~N}$ dando como resultados la concentración de esfuerzos en sus zonas con mayor tensión 1.5 Mpa., los cuales están por debajo del módulo de plasticidad del material.

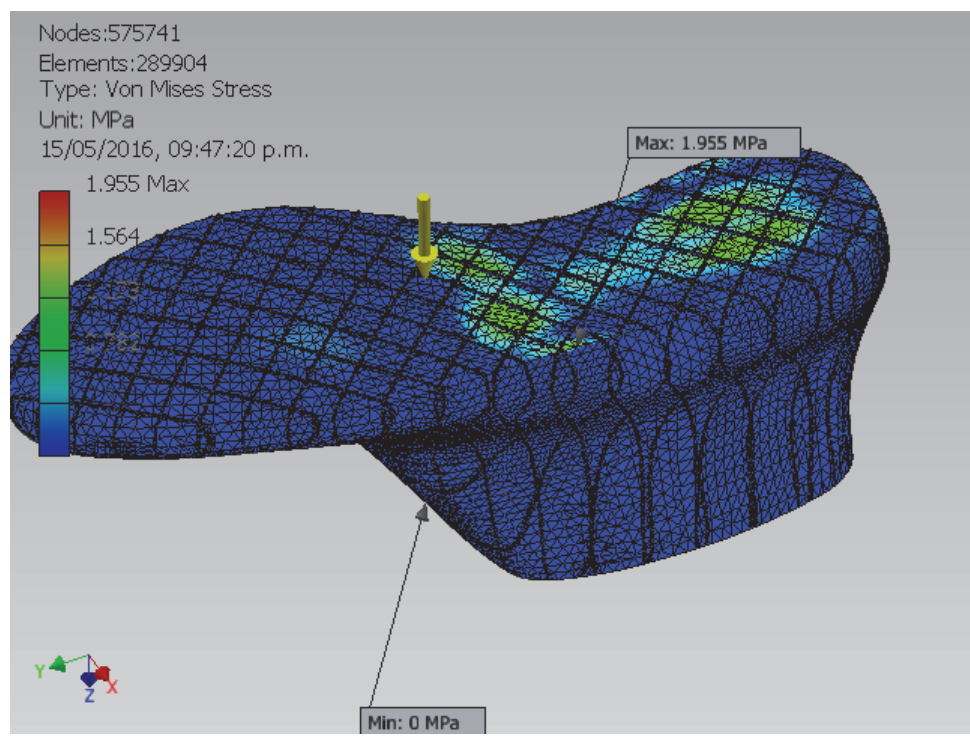

Fig. 8 Resultados de evaluación FEA en Inventor 2016- Autodesk. Fuente: Elaboración propia (2016)

La impresión de la horma se realizó mediante la utilización de un equipo de impresión 3D de modelado por deposición fundida (FDM por sus siglas in inglés) marca Airwolf, con velocidad de impresión de 25 $\mathrm{mm} / \mathrm{s}$, temperatura de cabezal de $240^{\circ} \mathrm{C}$ y de la cama de impresión de $115^{\circ} \mathrm{C}$. El espesor de capa de .25 $\mathrm{mm}$. y un espesor de pared de $0.5 \mathrm{~mm}$. El tiempo de elaboración fue de 7.3 horas consumiendo un total de 144 gramos de material.

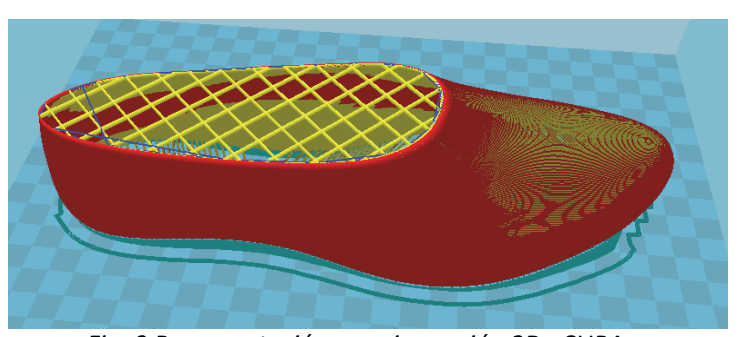

Fig. 9 Representación para impresión 3D. CURA V2.0 Fuente: Elaboración propia (2016)

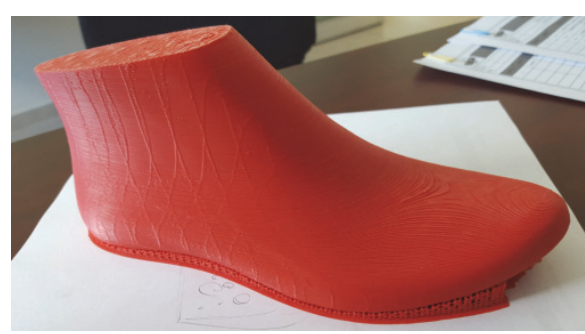

Fig.10 Horma impresa en 3D. Fuente: Elaboración propia (2016) 
Desarrollo de proceso para elaboración de horma personalizada mediante el uso de herramientas de manufactura flexible. Una visión sistémica,

\section{Resultados}

Obtención de par de hormas personalizadas para niña de 9 años con PEVAC, elaboradas de polímero ABS mediante el uso de impresión 3D, cuya estructura y características técnicas del material, permitirá su uso para la eventual fabricación de calzado en una empresa del ramo con equipos industriales con presiones de hasta 1.5 Bares.

\section{Conclusiones}

Una vez seleccionado el tipo de diseño de horma en base a las preferencias estéticas de la menor atendida, se procedió a la adecuación de la geometría en base a las dimensiones de sus pies. En este sentido, para el desarrollo de la geometría de la horma, sería en extremo útil la existencia de un sistema como el equipo YETI 3D scanner de la marca VORUM (VORUM, 2016) que permitiera la captura de las dimensiones de las extremidades con PEVAC adaptándolas a las hormas ya existentes, con la finalidad de generar con mayor rapidez el fichero para impresión en 3D. De esta manera se podrían potencializar dos herramientas para la generación más expedita de las hormas atacando a un nicho de mercado hoy no atendido.

\section{Trabajos futuros}

A partir de la obtención de la geometría del pie de la menor con PEVAC para la fabricación de su horma personalizada, hay la posibilidad de llevar a cabo un proceso similar para la elaboración de plantillas, ya que en la actualidad, hay materiales imprimibles en 3D con las características de flexibilidad y comodidad que permitirían su uso cotidiano.

\section{Referencias}

ADIDAS. (7 de Octubre de 2015). adidas breaks the mould with 3D-printed performance footwear. Recuperado el 12 de Mayo de 2016, de http://www.adidas-group.com/en/media/news-archive/press-releases/2015/adidas-breaksmould-3d-printed-performance-footwear/

DELCAM. (19 de marzo de 2010). Novedades. Recuperado el 12 de mayo de 2016, de Delcam to launch new 3d Scaner to orthotic design on ortopaedie: http://www.delcam.com/news/press_article.asp?releaseId=887

DOBBS MB, G. C. (2009). Update on clubfoot: etiology and treatment. . Clin Orthop Relat Res , 467 (5): 11461153.

ARMANDO TORRES-GÓMEZ, D. D.-S.-M.-Z. (2010). Pie equino varo aducto congénito,. Revista Mexicana de , $15-18$.

GEOBEATS. (2012). Shoes Created Using 3D Printer. Recuperado el 12 de mayo de 2016, de youtube.com: https://www.youtube.com/watch?v=9bZ7QE08ej0

HEIJNEN, L. (2004). World federation of hemophilia. Recuperado el 12 de 05 de 2016, de World federation of hemophilia: www1.wfh.org/publication/files/pdf-1110.pdf/

INFOWORK. (2014). infoworktecnology. Recuperado el 12 de mayo de 2016, de mpresión 3D viene con fuerza para revolucionar nuestro modo de vida.: http://www.infowork.es/impresion-3d-para-2014.html

KERR, D. (10 de junio de 2014). Los soportes ortopédicos de impresión 3D son más elegantes y cómodos. Recuperado el 12 de mayo de 2014, de Cnet.com: http://www.cnet.com/es/noticias/los-soportes-ortopedicos-deimpresion-3d-son-mas-elegantes-y-comodos/ 
MATTHEW B. DOBBS, C. A. (2009). Update on Clubfoot: Etiology and Treatment. Clinical Orthopaedics and Related Research , 467 (5): 1146 - 1153.

PÉLISSIER, A. B. (1 de enero de 2004). Zapatos ortopédicos. Recuperado el 12 de mayo de 2016, de Zapatos ortopédicos: http://www.em-consulte.com/es/article/40637/zapatos-ortopedicos

PRINT.COM, 3. (15 de 01 de 2016). Inside 3D printing. (innorobo.com, Productor) Recuperado el 28 de 04 de 2016, de 3D Print.com: https://3dprint.com/111713/70-yr-old-shoemaker-3d-print/

SLS. (2014). Shioeo Last Shop: <http://shoe-last-shop.com>. [Recuperado el 13 de mayo de 2016]

STAHELI L.T. (2006). Pie Zambo. Ortopedi Pediátrica, Madrid (España), Capítulo 5, 102 - 109.

THE SNEAKER FACTORY. How Shoes are Made -. (01 de junio de 2015). Recuperado el 13 de 05 de 2016, de Download Shoe Last for 3D Printing: http://sneakerfactory.net/sneakers/2015/06/download-shoe-last-for-3d-printing

VÁZQUEZ VG. (1987). Anomalías congénitas de pie. Pie equino varo congénito;. En Limusa (Ed.), Deformidades del pie, tratamiento conservador (Vol. 1a ed., págs. 227 - 234). México D.F. , Mèxico

VÄZQUEZ VG (1987). Deformaciones del pié, tratamiento conservador. México D.F.: Limusa.

VIJAYARAGAVAN, E. L. (2014). Application of Rapid Prototyping in the treatment of clubfoot in children. En ScienceDirect (Ed.), 12th GLOBAL CONGRESS ON MANUFACTURING AND MANAGEMENT, GCMM 2014 (págs. 2298 - 2305). ScienceDirect.

VORUM. (2016). VORUM. (VORUM, Productor), de Escáner 3D YETI: http://vorum.com/es/footwear/yeti-3d-footscanner/ [Recuperado el 03 de 05 de 2016]. 